\title{
Lesiones deportivas en la selección mexicana de clavados entre los Juegos Centroamericanos y del Caribe 2018, y los Juegos Panamericanos 2019
}

\section{Sports injuries in mexican diving team between the Central American and Caribbean Games 2018, and Pan American Games 2019}

\author{
Estela Labastida-Espinoza ${ }^{1}$ (iD
}

${ }^{1}$ Fisioterapeuta, Comisión Nacional de Cultura Física y Deporte. Ciudad de México, México. stella.labastida@gmail.com

Cómo citar: Cómo citar: Labastida-Espinoza, E. 2021. Lesiones deportivas en la selección mexicana de clavados entre los Juegos Centroamericanos y del Caribe 2018, y los Juegos Panamericanos 2019. Rev. Digit. Act. Fis. Deport. 7(2):e1831. http://doi.org/10.31910/rdafd. v7.n2.2021.1831

Artículo de acceso abierto publicado por Revista Digital: Actividad Física y Deporte, bajo una licencia Creative Commons CC BY-NC 4.0

Publicación oficial de la Universidad de Ciencias Aplicadas y Ambientales U.D.C.A, Institución de Educación Superior Acreditada de Alta Calidad por el Ministerio de Educación Nacional.

Recibido: febrero 17 de 2021 Aceptado: abril 13 de 2021 Editado por: Álvaro José Gracia Díaz

\section{RESUMEN}

Introducción: Actualmente, pocos estudios existen sobre la frecuencia de lesiones deportivas en la disciplina de clavados en México, a pesar que podrían ser útiles para desarrollar protocolos, que ayuden a disminuir los factores de riesgo en esta práctica. Objetivo: El propósito de esta investigación es determinar la frecuencia de lesiones deportivas en el equipo de primera fuerza de la selección mexicana de clavados, durante el periodo comprendido del 14 de junio de 2018 al 14 de agosto del 2019. Metodología: Se realizó un análisis cuantitativo, retrospectivo, longitudinal y descriptivo, de los datos del equipo de primera fuerza de la selección mexicana de clavados, a través del estudio de la frecuencia con el programa Excel y la representación gráfica de la información obtenida. Resultados: En el periodo examinado, se registraron 74 lesiones en total $(n=74)$ : el $84 \%$, no fueron traumáticas y el $74 \%$ ocurrieron durante el entrenamiento (62 y 55 incidencias, respectivamente). Con relación a la frecuencia y el tipo de lesiones, el 51\%, se ocasionaron en la prueba olímpica del trampolín de $3 \mathrm{~m}$; el $22 \%$, afectaron la zona anatómica del hombro y el $34 \%$, al tejido tendinoso. La mayoría de los casos, se presentaron en atletas de género masculino (57\%). Conclusiones: Los resultados obtenidos concuerdan con la incidencia descrita en la literatura internacional y la información sobre la frecuencia de lesiones en clavadistas mexicanos, podría servir como referencia para futuros análisis, pero se sugiere emplear una metodología rigurosa para el proceso de recolección de datos, seleccionar una muestra mucho más amplia y definir un periodo de tiempo mayor.

Palabras clave: Clavados; Lesión deportiva; Estadística; Competición; Entrenamiento; Incidencia; Frecuencia; Epidemiologia; Lesión músculo esquelética; Prevalencia.

\section{ABSTRACT}

Introduction: Currently there are few studies on the frequency of sports injuries in the diving discipline in Mexico, though they could be used in the development of protocols that help to reduce risk factors in this practice. Objective: The purpose of this research is to determinate the frequency of sports injuries in the Mexican diving team, during the period from June 14, 2018 to August 14, 2019. Methodology: 
A quantitative, retrospective, longitudinal and descriptive data of the team, through the study of the frequency with the Excel program and the graphic representation of the information obtained. Results: In the period examined, there were 74 injuries in total $(n=74)$ : $84 \%$ of them were non-traumatic and $74 \%$ occurred during training (62 and 55 incidents, respectively). Regarding the type of injuries, 51\% were caused in the Olympic test of 3-meter diving board, $22 \%$ affected the anatomical area of the shoulder and $34 \%$ the tendon tissue. Most of the cases occurred in male athletes (57\%) and in those with less sporting experience (58\%). Conclusions: The results obtained in this study are consistent with the incidence described in the international literature, and the information on the frequency of injuries in Mexican divers could be used for future studies, but use a rigorous methodology for the data collection process, select a bigger sample and define a longer period of time would be recommended.

Keywords:

Statistics;

Frequency; Epidemiology; Skeletal muscle injury; Prevalence.

\section{INTRODUCCIÓN}

Esta investigación busca determinar la frecuencia de lesiones deportivas en el equipo de primera fuerza de la selección mexicana de clavados, debido a la falta de estudios epidemiológicos al respecto; de esta manera, se podría contribuir con el desarrollo de futuros protocolos de prevención, que ayuden a disminuir los factores de riesgo de lesiones, durante la actividad física o la práctica deportiva.

La disciplina de clavados consiste en saltar desde un trampolín o plataforma, realizando vueltas y giros en el aire, para después entrar al agua. Existen dos métodos de entrada: con los pies o con las manos. En competencias es común que los clavadistas utilicen el segundo, para evitar salpicaduras y así obtener mejor puntaje, pero es importante considerar que, si no se realiza con precisión, la fuerza aplicada repercute en la fisonomía de las manos, los brazos e, incluso, el cuello y la columna (Haase, 2017).

Por lo anterior, el desarrollo de habilidades kinestésicas y propioceptivas, además de una gran fuerza física y elasticidad, resulta imprescindible para que los atletas puedan realizar acrobacias complejas, durante el clavado (Steinbrück \& Paeslack,1980).
En esta disciplina, el riesgo de lesiones deportivas es alto y muchas de ellas pueden ser mortales; sin embargo, la incidencia y la gravedad se reducen considerablemente, si los clavados se ejecutan por atletas de alto rendimiento, con conocimiento de las mecánicas y las técnicas para entrar al agua (Badman \& Rechtine, 2004). Como su nombre lo indica, las lesiones deportivas se asocian a la práctica de actividad física o algún deporte, que impiden continuar con el entrenamiento o la competencia de forma normal (Watson, 1997); su clasificación depende de las consecuencias inmediatas y posteriores sobre la salud física del atleta. Las repercusiones son variadas, van desde reducir la carga y mantenerse en completa inactividad por lapsos variables de tiempo, hasta abandonar, de manera voluntaria, el deporte, sufrir una discapacidad motriz o la muerte.

La literatura internacional sobre epidemiología de lesiones deportivas en la disciplina de clavados es escasa y poco clara sobre su frecuencia. Algunos autores, se enfocan en su relación con la biomecánica del clavado, mientras que otros realizan un análisis de datos y examinan su incidencia en zonas comunes. En los siguientes párrafos, se ofrece un panorama general sobre estos trabajos, con el fin de proporcionar un marco de referencia.

Rubin (1999), por ejemplo, considera que la columna torácica y lumbar son regiones comunes en las que se presentan lesiones en la práctica de clavados, debido a la flexión e hiperextensión forzada y rápida del tronco, lo cual, genera un aumento de la carga en el segmento anterior y posterior del cuerpo vertebral y provoca patologías discales. Además, los constantes microtraumas en el hombro del clavadista generan diferentes afectaciones, como lesión labral de anterior a posterior (SLAP por sus siglas en idioma inglés), lesión de Bankart o tendinopatía del supraespinoso.

Sobre esta misma área, Baranto et al. (2006) realizaron un estudio de 5 años de la columna vertebral de jóvenes clavadistas de élite, con seguimiento de resonancia magnética. Al principio, el $67 \%$ de los atletas presentaron un proceso degenerativo en la columna, pero durante el seguimiento, la cifra aumentó a $89 \%$, por lo que concluyeron que, este tipo de clavadistas, padece dolor de espalda a edades tempranas y corre mayor riesgo de desarrollar anormalidades degenerativas de la columna toracolumbar, probablemente, a causa de las lesiones. 
Por su parte, Narita et al. (2014) también analizaron, durante varios años (2003 a 2011, con excepción de 2006), a 83 clavadistas japoneses, durante su entrenamiento para la Federación de Natación de su país, encontrando que el 37,3\% de los participantes, presentó dolor lumbar.
Otras partes del cuerpo, comúnmente afectadas, son el hombro, el codo, la muñeca y la mano. Haase (2017) compiló los datos de diversos estudios en las pruebas (trampolín o plataforma) y registró que la mayoría de lesiones en estas áreas, se deben a la presión ejercida en los miembros superiores al momento de entrar al agua (Tabla 1$)$.

Tabla 1. Lesiones en mano, muñeca, codo y hombro reportadas en la literatura.

\begin{tabular}{|c|c|c|}
\hline Autor & Tipo de prueba & Lesión \\
\hline Waninger (1997) & Plataforma & Fractura de estrés de clavícula. \\
\hline Shinozaki et al. 2006) & Plataforma & $\begin{array}{c}\text { Fractura de estrés del olecranon, lesiones } \\
\text { en el ligamento colateral medial e } \\
\text { inestabilidad que puede desarrollar de } \\
\text { neuritis cubital. }\end{array}$ \\
\hline Berkoff \& Boggess (2011) & Plataforma y trampolín & $\begin{array}{l}\text { Múltiples contusiones óseas (semilunar, } \\
\text { capitate, hamate, radio parte distal) y } \\
\text { desgarro periférico del Complejo } \\
\text { Triangular del Fibrocartílago (TFCC). }\end{array}$ \\
\hline Haflah et al. (2014) & Plataforma & $\begin{array}{c}\text { Fractura de estrés de escafoides } \\
\text { bilaterales. }\end{array}$ \\
\hline Hosey et al. (2006) & Plataforma y trampolín & Fractura de estrés de escafoides. \\
\hline Le Viet et al. (1993) & Plataforma & $\begin{array}{c}\text { Microfractura de estiloides radial, } \\
\text { perforación del complejo del fibrocartílago } \\
\text { triangular y pseudoartrosis del hueso } \\
\text { hamate. }\end{array}$ \\
\hline Dawson (1992) & Trampolín & Ruptura de extensor largo del pulgar. \\
\hline
\end{tabular}

Fuente: Haase (2017).

Además de los tipos que se observan, una lesión común en la muñeca es la disrupción del ligamento escafolunar, que se presentó en el $78 \%$ de los clavadistas de una muestra (Beck, 2020).

Es importante destacar que los estudios realizados durante competencias aportan información valiosa en torno al total de lesiones y los tipos más frecuentes. En el campeonato mundial de la Federación Internacional de Natación (FINA) 2009, Mountjoy et al. (2010) reportaron 171 lesiones, lo que significó una incidencia de 66,0 por 1.000 atletas registrados. Las partes del cuerpo principalmente afectadas fueron el hombro $(n=25 ; 14,6 \%)$ y la cabeza $(n=21$; $12,3 \%$ ). La mitad de las lesiones ocurrieron durante el entrenamiento y la causa más común de lesión fue el uso excesivo ( $n=61 ; 37,5 \%$ ). La mayor incidencia de lesiones entre los atletas, se observó en la disciplina de los clavados, mientras que la más baja, fue en natación.

Años después, en los campeonatos mundiales FINA 2013, se evidenció que las lesiones más frecuentes de la parte inferior del cuerpo ocurren en las rodillas (Mountjoy et al. 2015). En esta misma línea Vieira et al. (2017) realizaron un estudio con atletas élite de clavados, utilizando el método isocinético, para examinar la fuerza de los flexores y extensores de la rodilla; observaron que los músculos extensores tienen mayor fuerza que los músculos flexores, lo que explica la mayoría de las lesiones en estas áreas.

En la investigación de Kerr et al. (2015), se analizaron las lesiones de clavadistas reportadas por la National Collegiate Athletic Association (NCAA), durante el periodo académico 2009-2010 a 2013-2014. De 
acuerdo con estos autores, se presentaron 149 lesiones por natación y clavados, lo que se traduce en tasas de incidencia de 1,54 y 1,71 por cada 1.000 atletas registrados en estas disciplinas. Las mujeres clavadistas sufrieron más lesiones (2,49 por 1.000), en comparación con los hombres (1,94 por 1.000); las más frecuentes ocurrieron en el hombro, provocaron distensiones y se clasificaron como lesiones por uso excesivo o sin contacto. La incidencia de estos casos fue de 0,54 por 1.000 , en mujeres y de 0,46 por 1.000 , en hombres. Durante los periodos 2012, 2013-2013 y 2014 hubo menos incidencias que a lo largo de 2009, 2010-2011 y 2012
Ahora bien, el caso de los Juegos Olímpicos merece particular atención, porque en Río de Janeiro 2016, se registraron solo 12 lesiones entre atletas de clavados y ninguna de las ellas impidió que continuaran con sus actividades deportivas (Soligard et al. 2017). En Londres 2012, la incidencia fue similar (11 casos en total) (Engebretsen et al. 2013). Los datos sobre el tipo y la zona donde se ocasionaron las lesiones, en ambas competencias, se muestran en la tabla 2.

Tabla 2. Localización y tipo de lesiones en clavadistas durante los Juegos Olímpicos de Verano Río de Janeiro, en el 2016.

\begin{tabular}{|l|c|}
\hline Localización & Incidencia \\
\hline Cara & $(1 / 12)$ \\
\hline Abdomen & $(1 / 12)$ \\
\hline Columna lumbar & $(1 / 12)$ \\
\hline Hombro & $(1 / 12)$ \\
\hline Brazo & $(1 / 12)$ \\
\hline Cadera & $(1 / 12)$ \\
\hline Muslo & $(1 / 12)$ \\
\hline Rodilla & $(2 / 12)$ \\
\hline Pierna & $(1 / 12)$ \\
\hline Pie & $(2 / 12)$ \\
\hline Tipo & Incidencia \\
\hline Contusión/ hematoma & $(1 / 12)$ \\
\hline Pinzamiento subacromial & $(1 / 12)$ \\
\hline Esguince & $(3 / 12)$ \\
\hline Lesión muscular & $(2 / 12)$ \\
\hline Tendinopatía & $(2 / 12)$ \\
\hline Otra & $(3 / 12)$ \\
\hline
\end{tabular}

El objetivo del presente trabajo fue determinar la frecuencia de lesiones deportivas en el equipo de primera fuerza de la Selección Mexicana de Clavados, en específico, detectar si las lesiones fueron o no traumáticas y cuáles tuvieron mayor incidencia, detallar la zona anatómica y el tipo de tejido con mayor frecuencia de lesiones, establecer la prueba de clavados con mayor incidencia de lesiones y caracterizar el momento de lesión con mayor incidencia.

\section{MATERIALES Y MÉTODOS}

Por su enfoque metodológico, esta investigación es de carácter cuantitativo, retrospectivo, longitudinal y descriptivo. El análisis, se realizó con el equipo de primera fuerza de la selección mexicana de clavados, en las instalaciones deportivas de la Ciudad de México, Guadalajara y las sedes de competencia, durante el periodo del 14 de junio del 2018 hasta el 14 de agosto del 2019. La población del estudio fue de 60 atletas y la población de muestra fue de 20 participantes: 12 hombres y 8 mujeres, con edad promedio de 23 años (rango, 15 a 34 años, DE \pm 5), talla promedio de $1,64 \mathrm{~cm}$ (DE \pm 7 ), peso promedio de $60 \mathrm{~kg}(\mathrm{DE} \pm 7)$ e índice de masa corporal promedio de $21 \mathrm{~kg} / \mathrm{m}^{2}(\mathrm{DE} \pm 1)$. 


\section{Criterios de inclusión:}

- Cumplir con los criterios establecidos por la Federación Mexicana de Natación (FMN, 2017), para formar parte del equipo de primera fuerza de la selección mexicana de clavados.

- Cumplir con los criterios establecidos por la FINA (2017), para participar en eventos internacionales.

- Tener, por lo menos, un año como seleccionado del equipo de primera fuerza de la selección nacional de clavados.

- $\quad$ Participar en las pruebas olímpicas (trampolín de $3 \mathrm{~m}$ y plataforma de $10 \mathrm{~m}$ ).

\section{Criterios de exclusión:}

- $\quad$ Presentar una lesión ocasionada por causas externas a la práctica deportiva de clavados, durante el periodo evaluado del estudio.

- Estar lesionado durante la evaluación inicial del estudio.

- Presentar lesiones deportivas que ameriten una baja menor a 24 horas.

- $\quad$ No querer participar en el estudio.

\section{Criterios de eliminación:}

- $\quad$ Dejar de formar parte del equipo de primera fuerza de la selección mexicana de clavados, durante el periodo comprendido entre el 14 de junio de 2018 al 14 de agosto de 2019.

- $\quad$ Abandonar el seguimiento y registro de datos.

El procedimiento para realizar el estudio comprendió tres fases. En la primera, se investigaron los antecedentes de clavados y de lesiones deportivas, en bases de datos, como PubMed, Scopus, Redalyc, EBSCO, Scholar y Ébano; se utilizaron las siguientes palabras clave: lesiones deportivas, lesiones músculoesqueléticas, historia de clavados, estadística de lesiones en clavados, estadística de lesiones deportivas en juegos olímpicos, incidencia y prevalencia de lesión.
En la segunda fase, se evaluaron a los atletas para verificar que no presentaran lesiones y se registraron los datos de edad, peso, talla e índice de masa corporal, en un formato estandarizado. La recolección de estos datos duró 12 meses y se realizó en las diferentes sedes donde entrenó y participó el equipo de primera fuerza de la selección mexicana de clavados. Por último, en la tercera fase, se obtuvo información específica sobre las lesiones deportivas, la cual, se revió, procesó y analizó con el programa Excel, para poder interpretarlos y proyectarlos gráficamente.

\section{RESULTADOS Y DISCUSIÓN}

En total, se registraron 74 lesiones, en 2.464 horas de exposición, durante el periodo comprendido entre el 14 de junio de 2018 al 14 de agosto de 2019, repartidas en 2.088 (84\%) horas de entrenamiento y $376(n=16 \%)$ horas de competencia.

Los equipos reportaron que cada clavadista realizó en promedio 131 sesiones de entrenamiento y 5 competencias durante el período de revisión.

Se observó, que el mayor número de lesiones se presentaron durante el entrenamiento $(n=55 ; 74 \%)$ en comparación con las lesiones durante competencia $(n=19 ; 26 \%)$.

Se obtuvo menor incidencia en las lesiones de tipo traumáticas $(n=12 ; 16 \%)$, en comparación a las lesiones no traumáticas ( $n=62 ; 84 \%)$.

En la figura 1a se puede ver la distribución relativa de las diferentes zonas anatómicas de lesión y su frecuencia. El hombro fue la zona anatómica con mayor incidencia ( $n=16 ; 22 \%)$, seguida de la rodilla $(n=15 ; 20 \%)$ y en tercer lugar el muslo ( $n=9 ; 12 \%)$.

La distribución de las lesiones por tipo de tejido, siendo el tendón el tejido más afectado $(n=25 ; 33.8 \%)$, seguido por las lesiones articulares ( $n=15 ; 20,3 \%)$, se muestra en la figura $1 b$.

De acuerdo con la prueba olímpica el 51\% ( $n=38)$ de las lesiones se presentó en la prueba de trampolín de 3 metros, mientras que el $49 \%(n=36)$, en la prueba de plataforma de 10 metros. 
(a)

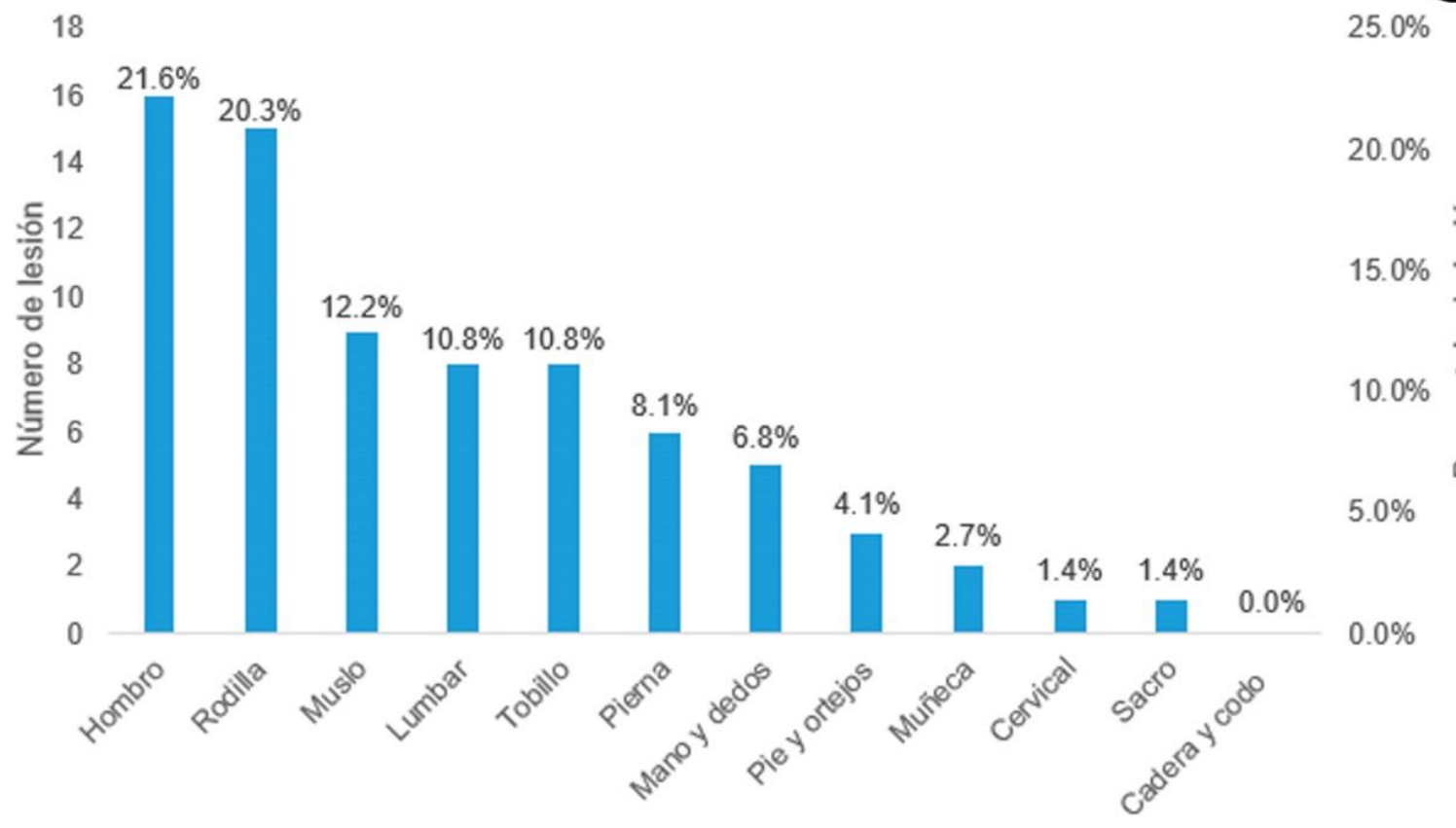

Zona anatómica

30

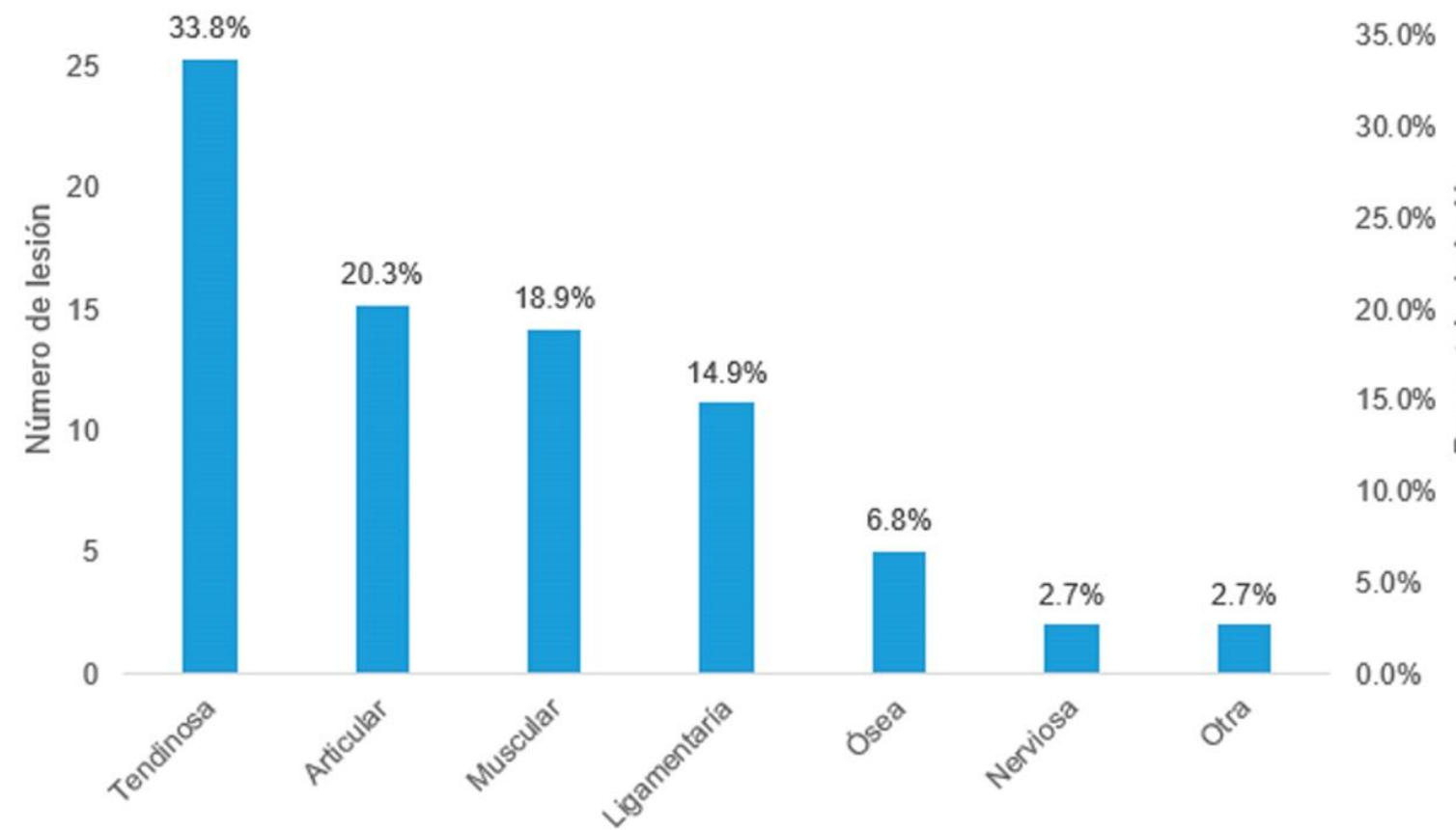

Tipo de tejido

Figura 1. Porcentaje de lesiones en la Selección Mexicana de Clavados, durante el periodo comprendido del 14 de junio de 2018 al 14 de agosto de 2019. a. por zona anatómica; b. por tipo de tejido.

Del total de las lesiones presentadas en la lado, del total de las lesiones presentadas en la prueba de $10 m, 41,7 \%(n=15)$ correspondieron a prueba de $3 m, 28,9 \%(n=11)$ correspondieron a lesiones tendinosas; $16,7 \%(n=6)$, a musculares y lesiones articulares; $26,3 \%(n=10)$, a tendinosas y el el 13,9\% ( $n=5)$, a ligamentos (Figura 2a). Por otro 21,1 \% ( $n=8)$, a musculares (Figura $2 b)$. 

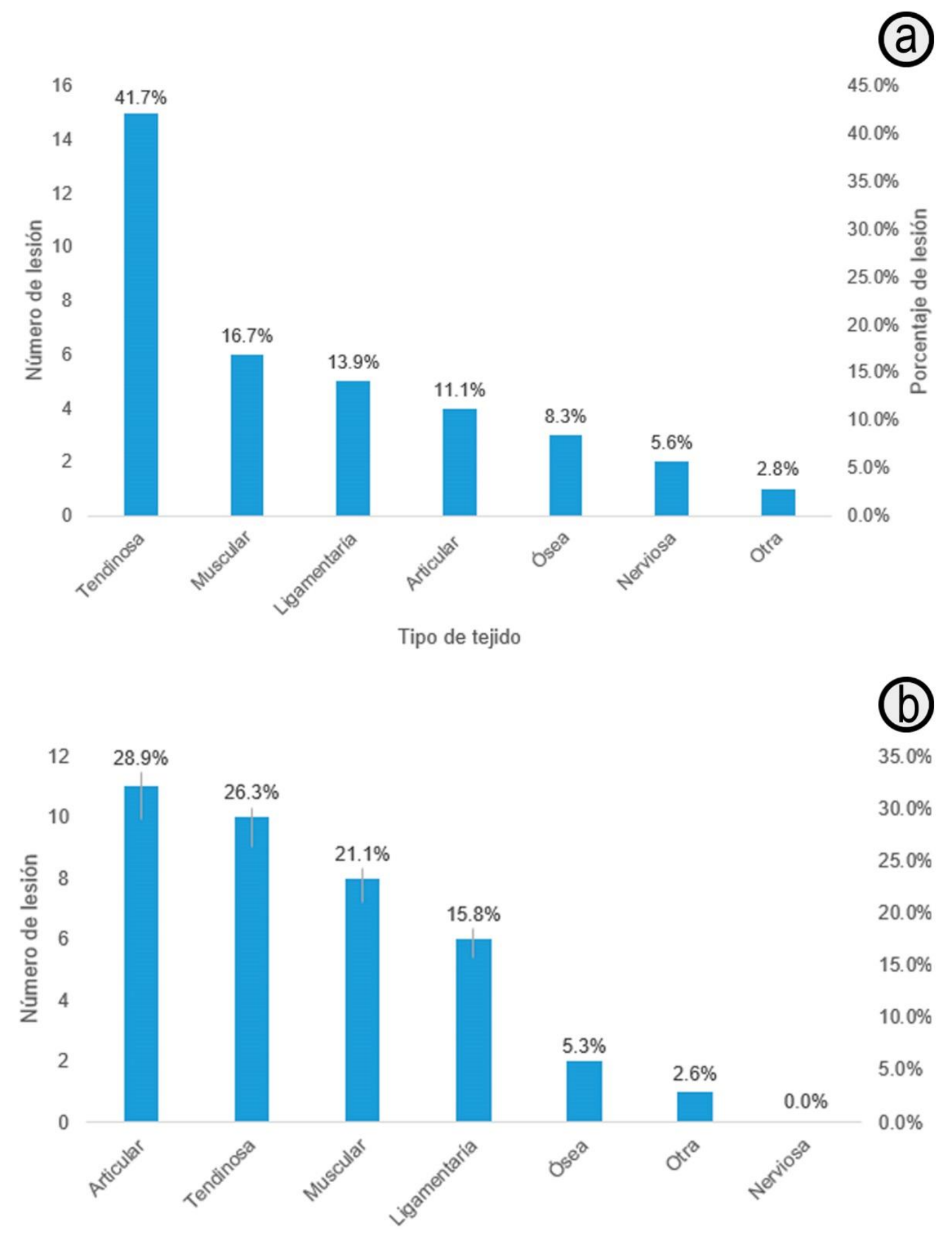

Tipo de tejido

Figura 2. Porcentaje de lesiones por prueba de la Selección Mexicana de Clavados, durante el periodo comprendido del 14 de junio de 2018 al 14 de agosto de 2019. a. plataforma 10m; b. trampolín 3m.

De acuerdo con el género, la rama varonil tuvo 43 a musculares (Figura 3a). Finalmente, del total de (57\%) casos de lesiones, mientras que la rama femenil las lesiones presentadas en la rama varonil fue tuvo $32(43 \%)$ casos.

$35,7 \% \quad(n=15) \quad$ correspondieron a lesiones tendinosas; $21,4 \% \quad(n=9)$, a ligamentos; $14,3 \%$

Del total de las lesiones presentadas en la rama $(n=6)$, a articulares y el $14,3 \%(n=6)$, a musculares femenil, 31,3\% ( $n=10)$ correspondieron a lesiones (Figura 3b).

tendinosas; $28,1 \%(n=9)$, a articulares y el $25 \%(n=8)$, 
(a)

12 $31.3 \%$
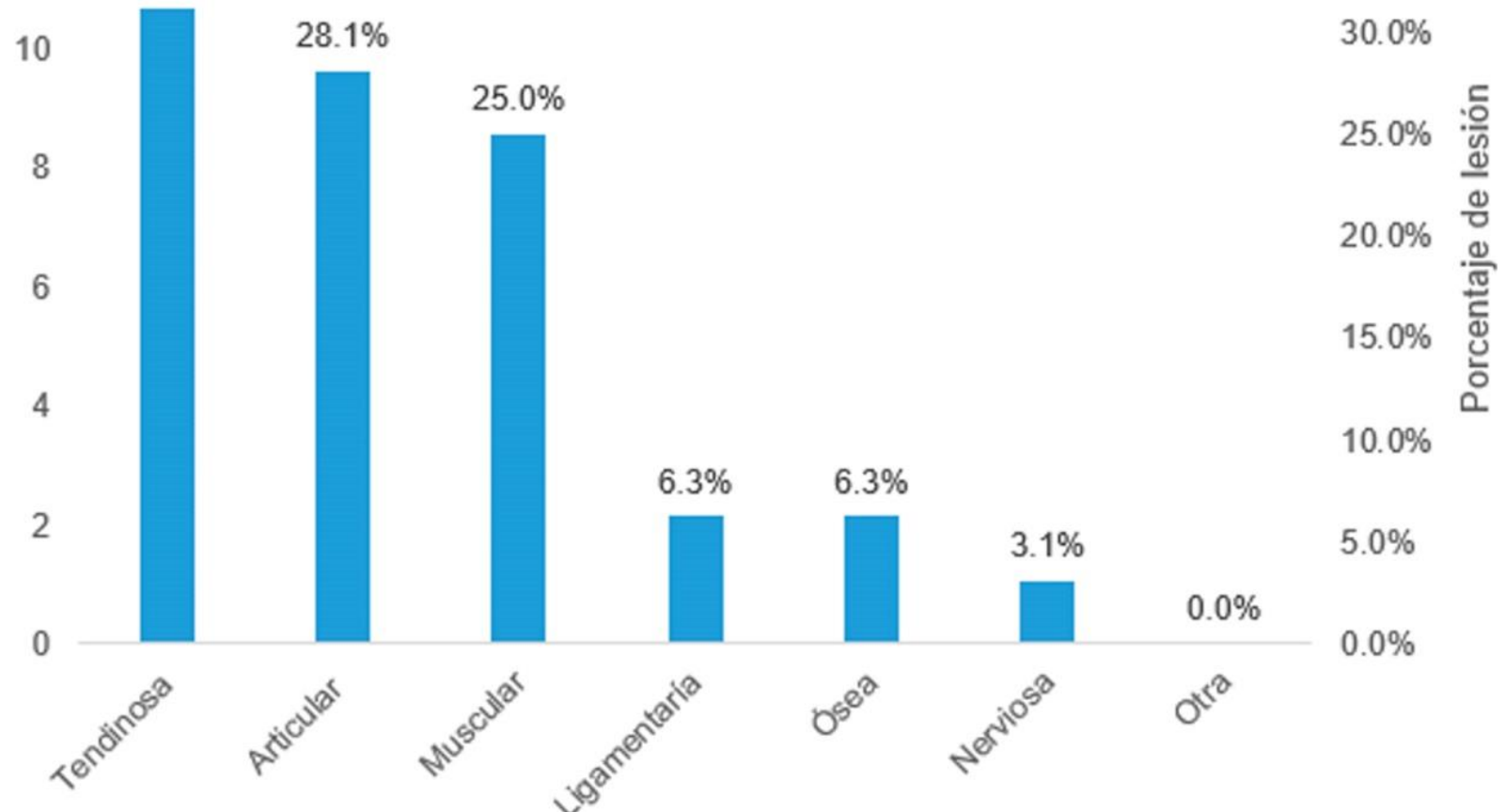

Tipo de tejido

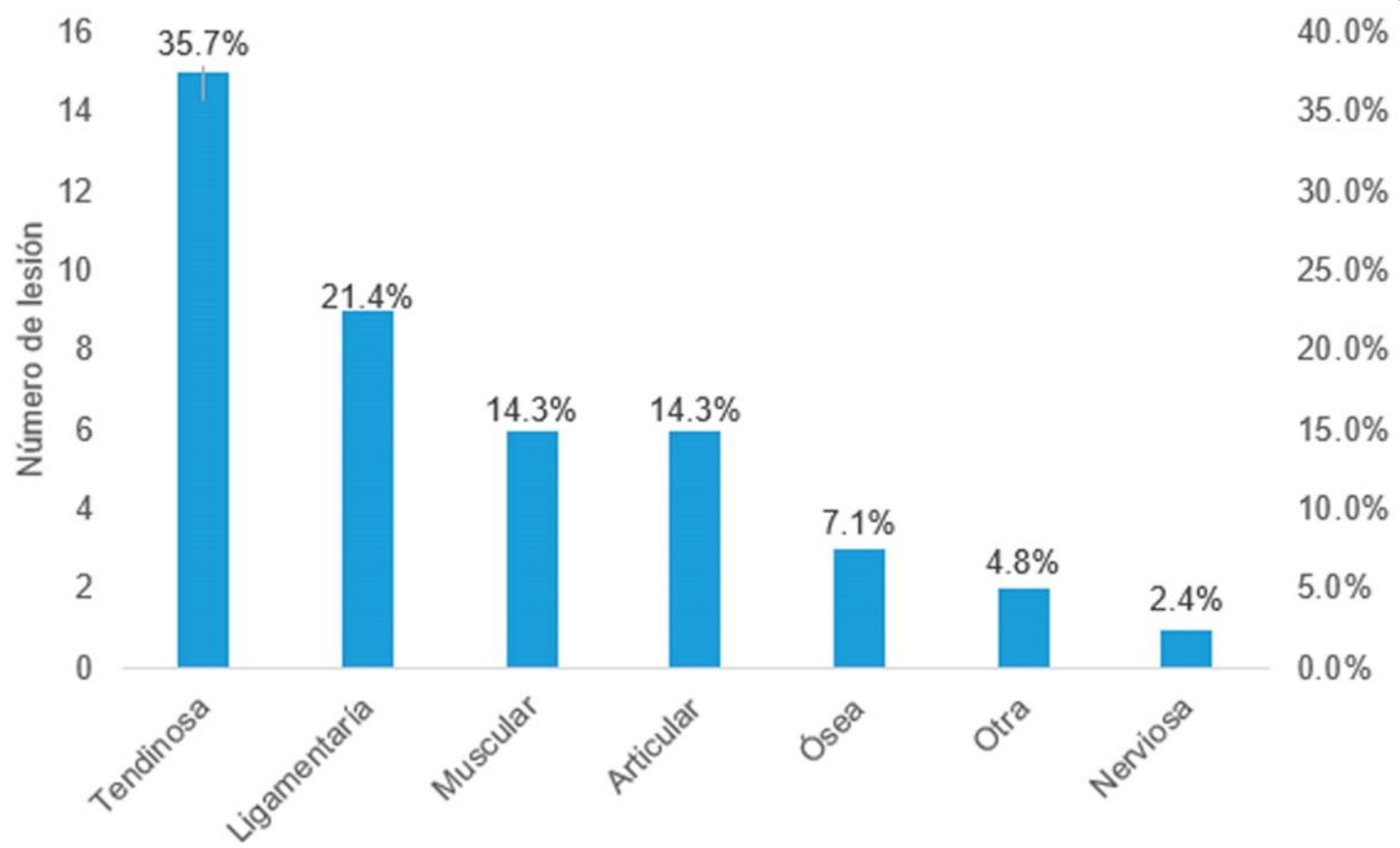

Figura 3. Porcentaje de lesiones por género en la de la Selección Mexicana de Clavados, durante el periodo comprendido del 14 de junio de 2018 al 14 de agosto de 2019. a. rama femenil; b. rama varonil. 
El registro de lesiones de los atletas de la selección mexicana de clavados, recopilado durante el 14 de junio del 2018 al 14 de agosto del 2019, permite identificar y contrastar las siguientes variables: el momento de la lesión, el tipo (traumáticas o no), el tejido músculo esquelético y la zona del cuerpo afectados, así como la frecuencia entre los clavadistas profesionales.

El momento de lesión más frecuente reportado en este estudio es el entrenamiento, que coincide con los reportes de la literatura internacional y los resultados que aportan Mountjoy et al. (2010), en su estudio durante el campeonato mundial FINA 2009, aunque gran parte de las investigaciones, como el compilado de Haase (2017), no aclaran si las lesiones ocurren en un periodo de competencia o de entrenamiento.

Las lesiones sin contacto tienen mayor incidencia, lo que concuerda también con la literatura internacional y el estudio de Kerr et al. (2015), donde identifican al hombro, como la zona anatómica con mayor número de lesiones.

La segunda zona atómica con más casos de lesiones reportadas en la selección mexicana de clavados fue la rodilla, lo que se relaciona con los resultados de Vieira et al. (2017) y Mountjoy et al. (2015), durante los campeonatos mundiales FINA 2013.

En cuanto al tipo de lesión clasificada por tejido, el estudio de Rubin (1999), basado en la biomecánica del clavado, menciona que el hombro es una de las zonas anatómicas con mayor probabilidad a lesionarse y como el tendón resulta afectado, se produce tendinopatía del supraespinoso, pero su estudio no brinda la frecuencia o incidencia de este tipo de lesión.

Para obtener las estadísticas de lesiones deportivas, principalmente en los clavados, hace falta una clasificación estandarizada, que se pueda utilizar para complementar y contrastar estudios, como los de Soligard et al. (2017), contra estudios locales. Uno de los problemas para establecer una clasificación estándar en las lesiones deportivas está relacionado con la poca precisión para determinar el dolor que causa una lesión.

Al realizar esta investigación, se relacionan diversas dificultades, como una muestra de estudio pequeña, un periodo de tiempo corto, la coincidencia en los diagnósticos entre el personal médico y el inadecuado llenado del registro. Determinar el origen de las lesiones no fue el objetivo de este estudio; sin embargo, un estudio complementario que relacione las lesiones con las variables específicas de los atletas, como edad, peso, talla, IMC y género, ayudaría a establecer correlaciones que se puedan emplear para la detección y la prevención de estos padecimientos.

\section{CONCLUSIONES}

Con base en los resultados obtenidos es posible determinar la frecuencia de lesiones en clavadistas mexicanos, identificando mayor incidencia durante los entrenamientos, en la zona anatómica del hombro y, específicamente, en el tejido de tipo tendinoso. Estos datos concuerdan con lo que se establece en la literatura internacional y sirven como guía para generar protocolos de prevención y disminuir los factores de riesgo en esta disciplina deportiva; no obstante, se recomienda realizar un análisis profundo, con una metodología rigurosa en el proceso de recolección de datos, una muestra de estudio mucho más amplia y un periodo de tiempo mayor.

A pesar de las dificultades que se comentaron, el presente estudio cumple su objetivo de proporcionar datos, que sirvan como punto de comparación en futuras investigaciones, sobre la frecuencia de lesiones deportivas, en la población de la selección mexicana de clavados, además de ser una guía para el análisis de factores de riesgo.

\section{REFERENCIAS}

1. BADMAN, B.L.; RECHTINE, G.R. 2004. Spinal injury considerations in the competitive diver: $A$ case report and review of the literature. The Spine Journal. 4(5):584-590. https://doi. org/10.1016/j.spinee.2004.03.002

2. BARANTO, A.; HELLSTRÖM, M.; NYMAN, R.; LUNDIN, O.; SWÄRD, L. 2006. Back pain and degenerative abnormalities in the spine of young elite divers 5-year follow-up magnetic resonance imaging study. Knee Surgery, Sports Traumatology, Arthroscopy. 14:907-914. https://doi.org/10.1007/s00167005-0032-3

3. BECK, A. 2020. The incidence of scapholunate ligament disruption in competitive divers. British Journal of Sports Medicine. 54 (suppl. 1):A51-A51. 
4. ENGEBRETSEN, L.; SOLIGARD, T.; STEFFEN, K.; ALONSO, J.M.; AUBRY, M.; BUDGETT, R.; DVORAK, J.; JEGATHESAN, M.; MEEUWISSE, W.H.; MOUNTJOY, M.; PALMER-GREEN, D.; VANHEGAN, I.; RENSTRÖM, P.A. 2013. Sports injuries and illnesses during the London Summer Olympic Games 2012. British Journal of Sports Medicine. 47(7):407-414. https:// doi.org/10.1136/bjsports-2013-092380

\section{FEDERACIÓN INTERNACIONAL DE NATACIÓN, FINA.} 2017. Reglas de Clavados FINA. Disponible desde Internet en: https://www.fina.org/ content/fina-rules-regulations

6. FEDERACIÓN MEXICANA DE NATACIÓN, FMN. 2017. Reglamento Deportivo. Disciplina de Clavados. Disponible desde Internet en: http://fmn.org.mx/page/clavados

7. HAASE, S.C. 2017. Management. of upper extremity injury in divers. Hand Clinics. 33(1):73-80. https://doi.org/10.1016/j.hcl.2016.08.017

8. KERR, Z.; BAUGH, C.M.; HIBBERD, E.; SNOOK, E.; HAYDEN, R.; DOMPIER, T. 2015. Epidemiology of National Collegiate Athletic Association men's and women's swimming and diving injuries from 2009/2010 to 2013/2014. British Journal of Sports Medicine. 49:465-471. https://doi. org/10.1136/bjsports-2014-094423

9. MOUNTJOY, M.; JUNGE, A.; ALONSO, J.M.; ENGEBRETSEN, L.; DRAGAN, I.; GERRARD, D.; KOUIDRI, M.; LUEBS, E.; MORADI, F.; DVORAK, J. 2010. Sports injuries and illnesses in the 2009 FINA World Championships (Aquatics). British Journal of Sports Medicine. 44:522-527. https://doi.org/10.1136/bjsm.2010.071720

10. MOUNTJOY, M.; JUNGE, A.; BENJAMEN, S.; BOYD, K.; DIOP, M.; GERRARD, D.; VAN DEN HOOGENBAND, C.R.; MARKS, S.; MARTINEZRUIZ, E.; MILLER, J.; NANOUSIS, K.; SHAHPAR, F.M.; VELOSO, J.; VAN MECHELEN, W.; VERHAGEN, E. 2015. Competing with injuries: Injuries prior to and during the 15th FINA World
Championships 2013 (aquatics). British Journal of Sports Medicine. 49(1):37-43. https://doi.org/10.1136/bjsports-2014-0939 91

11. NARITA, T.; KANEOKA, K.; TAKEMURA, M.; SAKATA, Y.; NOMURA, T.; MIYAKAWA, S. 2014. Critical factors for the prevention of low back pain in elite junior divers. British Journal of Sports Medicine. 48:919-923. http://dx.doi. org/10.1136/bjsports-2012-091875

12. RUBIN, B. 1999. The basics of competitive Diving and its Injuries. Clinics in Sports Medicine. 18:296-302. https://doi.org/10.1016/ s0278-5919(05)70145-9

13. SOLIGARD, T.; STEFFEN, K.; PALMER, D.; ALONSO, J.M.; BARH, R.; DIAS LOPES, A.; DVORAK, J.; GRANT, M.E.; MEENWISSE, W.; MOUNTIOY, M.; PENA COSTA, L.O.; SALMINA, N.; BUDGETT, R.; ENGEBRETSEN, L. 2017. Sports injury and illness incidence in the Rio de Janeiro 2016 Olympic Summer Games: A prospective study of 11274 athletes from 207 countries. British Journal of Sports Medicine. 51(17):1265-1271. https://doi.org/10.1136/ bjsports-2017-097956

14. STEINBRÜCK, K.; PAESLACK, V. 1980. Analysis of 139 Injuries due to accidents in water sport. Paraplegia. 18(2):86-93. https://doi. org/10.1038/sc.1980.14

15. VIEIRA, A.; ALEX, S.; MARTORELLI, A.; BROWN, L.E.; MOREIRA, R.; BOTTARO, M. 2017. Lowerextremity isokinetic strength ratios of elite springboard and platform diving athletes. The Physician and Sports Medicine. 45:87-91. https://doi.org/10.1080/00913847.2017.130 2310

16. WATSON, A. 1997. Sports injuries: incidence, causes, prevention. Physical Therapy Reviews. 2(3):135-51. https://doi.org/10.1179/ ptr.1997.2.3.135 